\title{
The Impact of Disclosure of Intimate Partner Violence on Friends
}

\author{
BY \\ RANNVEIG SIGURVINSDOTTIR
}

B.S., University of Iceland, 2011

\author{
THESIS \\ Submitted as partial fulfillment of the requirements \\ for the degree of Master of Arts in Psychology \\ in the Graduate College of the \\ University of Illinois at Chicago, 2013
}

Chicago, Illinois

Defense Committee:

Stephanie Riger, Chair and Advisor

Sarah Ullman, Criminology Law and Justice

Paul Schewe, Criminology, Law and Justice 


\section{ACKNOWLEDGEMENTS}

I would like to thank my thesis committee, Stephanie Riger, Sarah Ullman and Paul Schewe, for their help and support with this project. I would also like to especially acknowledge Stephanie and Sarah for their unlimited support and guidance they have provided me with during my studies at UIC.

RS 


\section{TABLE OF CONTENTS}

CHAPTER

PAGE

$\mathrm{I}$

INTRODUCTION

A. Social reactions.......................................................... 2

B. Impact on Friends................................................. 2

C. Friends of IPV Survivors ........................................... 3

D. Current Study ....................................................... 4

II. METHOD ................................................................... 7

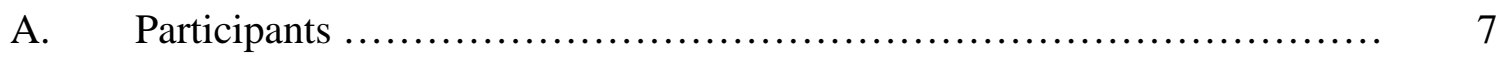

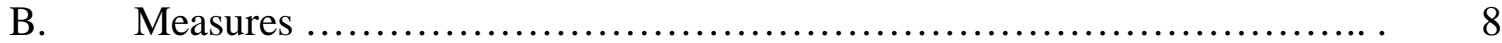

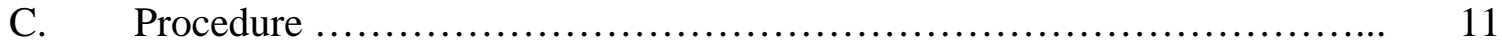

D. Analysis .............................................................. 12

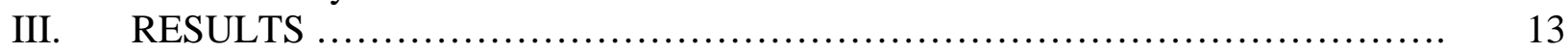

A. Descriptive Information of Impact on Friends-IPV measure................ 13

B. Relationship quality.................................................. 13

C. Gender............................................................. 14

D. Victimization..................................................... 14

E. Psychological symptoms.......................................... 14

F. Factor analysis..................................................... 16

G. Factor scores predicting psychological symptoms....................... 18

H. Mediated regression............................................... 18

I. Moderated regression............................................... 19

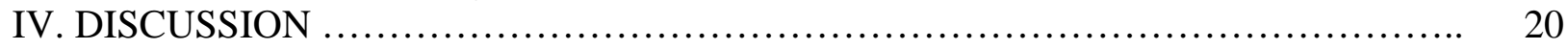

CITED LITERATURE....................................................... 26

INSTITUTIONAL REVIEW BOARD APPROVAL .......................... 42

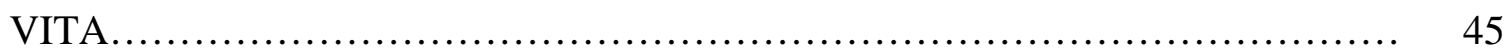




\section{LIST OF TABLES}

$\underline{\text { TABLE }}$

$\underline{\text { PAGE }}$

I. DEMOGRAPHICS FOR PARTICIPANTS INCLUDED AND EXCLUDED

FROM ANALYSIS AND DEMOGRAPHICS FOR FRIENDS AND NON-FRIENDS 33

II. NUMBER AND PERCENTAGES OF PARTICIPANTS WHO AGREED OR STRONGLY AGREED WITH EACH ITEM ON THE IMPACT ON FRIENDS SCALE .... 34 III. VICTIMIZATION DIFFERENCES ON THE IMPACT ON FRIENDS- IPV

MEASURE 35

IV. CORRELATION BETWEEN IOF ITEMS AND PSYCHOLOGICAL SYMPTOMS 36

V. PSYCHOLOGICAL SYMPTOMS BY VICTIMIZATION .............. 37 VI. STANDARDIZED FACTOR LOADINGS OF ITEMS AND HOW MUCH VARIANCE OF EACH ITEM THE FACTOR SOLUTION EXPLAINS .................. 38 VII. STANDARDIZED FACTOR LOADINGS OF ITEMS ................. 39 VIII. DESCRIPTIVE INFORMATION FOR IMPACT ON FRIENDS 


\section{LIST OF ABBREVIATIONS}

IPV Intimate partner violence

SA Sexual assault

IOF Impact on friends

PTSD Post-traumatic stress disorder 


\section{SUMMARY}

Intimate partner violence (IPV) affects others as well as the survivor (Riger, Raja \& Camacho, 2002), but we do not have measures to assess that impact. The Impact on Friends measure was developed to understand the impact of disclosure of sexual assault (SA) on friends of survivors (Ahrens \& Campbell, 2000; Banyard et al., 2010). In this study, the Impact on Friends measure was modified to apply to IPV and administered to an online sample. Exploratory factor analysis revealed four factors: Emotional Distress, Validation, Confusion and IPV. The first three factors are similar to the scale for friends of SA survivors (Banyard et al., 2010); a fourth factor refers specifically to IPV. The Impact on Friends-IPV measure has adequate reliability. One of its factors, Emotional Distress, predicts increased depression and post-traumatic distress disorder symptoms of friends. These results suggest that although there is overlap in the experience of hearing about assault or impact of that for both friends of IPV and SA survivors, disclosure of IPV has an additional, unique impact on friends. 


\section{INTRODUCTION}

Social support is important to survivors of intimate partner violence (IPV). In addition to help from professionals, such as doctors and shelter workers, survivors may seek help from informal supporters such as friends and family. They may help survivors leave abusive relationships or cope with IPV (El-Bassel, Gilbert, Rajah, Folleno \& Frye, 2001; Krishnan, Hilbert \& VanLeeuwen, 2001; Rose, Campbell \& Kub, 2000; Tan, Basta, Sullivan \& Davidson II, 1995; Thompson et al., 2000). Two-thirds of IPV survivors in the United States seek some form of informal support (Goodman, Dutton, Weinfurt \& Cook, 2003; Levendosky et al., 2004; Rose et al., 2000) and any form of social support is associated with decreased mental health problems (Campbell, Sullivan \& Davidson II, 1995; Carlson, McNutt, Choi \& Rose, 2002; Coker et al, 2002; Coker, Watkins, Smith and Brandt, 2003; El-Bassel et al., 2001; Tan et al., 1995; Thompson et al., 2000). Friends' reactions are especially salient to survivors, and positive reactions from friends are associated with better recovery (Campbell et al., 2001; Filipas \& Ullman, 2001; Ullman, 1996). Many survivors believe that informal support is more likely than support from formal agencies to end IPV because friends and family know the situation and the people involved (Budde \& Schene, 2004; Mancini, Nelson, Bowen \& Martin, 2006).

Despite the importance of informal support to survivors, little is known about how disclosure of IPV impacts others. Preliminary studies suggest a radiating impact of IPV on friends, family, neighbors and coworkers of the survivor (Riger et al., 2002). Sexual assault (SA) research suggests that the survivors' friends and family may experience distress and confusion about how to react to survivors (Ahrens \& Campbell, 2000). The Impact on Friends measure was developed to assess how disclosure of SA impacts friends of survivors (Ahrens \& Campbell, 2000; Banyard et al., 2010). We have no measure to assess the impact of disclosure of IPV on 
friends of survivors. In this study, the Impact on Friends measure was modified to understand how disclosure of IPV impacts friends, as well as to see whether the impact of IPV is similar to that of SA.

\section{Social reactions}

The term "social support" implies a positive impact, but others may not respond positively to a survivor (Ullman, 1999). Therefore, social support may be reconceptualized as social reactions. In SA research, positive reactions include emotional support and giving tangible aid, such as practical assistance or information. Negative reactions include blaming the survivor, trying to distract the survivor, treating the survivor differently than before, trying to control the survivor and becoming so distressed that the survivor feels guilty for disclosing violence (Ullman, 2000). Negative reactions from others have a bigger impact on health than positive reactions (Campbell, Ahrens, Sefl, Wasco \& Barnes, 2001) and are connected with poorer psychological and physical health of survivors as well as delayed recovery from SA (Ullman, 1996; Ullman \& Siegel, 1995). Less research exists on social reactions to IPV but preliminary studies suggest a detrimental effect of negative reactions and a limited impact of positive reactions on survivors (Goodkind, Gillum, Bybee \& Sullivan, 2003).

\section{Impact on Friends}

Little is known about how violence impacts those to whom survivors disclose violence. IPV may negatively affect friends, family, neighbors and coworkers of the survivor. For example, family members and friends may experience violence themselves from the perpetrator (Riger et al., 2002). This may explain the reluctance of some survivors to seek help and why others may choose to distance themselves from the survivor (Goodman et al., 2003; Riger et al., 
2002). Informal supporters are more likely to react negatively to survivors when the supporters have been physically threatened by the abuse (Goodman et al., 2003).

The impact of disclosure of SA depends on relationship to the survivor. For example, family members and romantic partners of survivors report negative emotions and interpersonal problems after disclosure (Ahrens \& Campbell, 2000; Davis \& Brickman, 1996). Also, friends, family members and romantic partners may be unsure of how to react to survivors (Ahrens \& Campbell, 2000). Friends, however, show fewer signs of distress and confusion than family members, though they may report shock, anger and wanting revenge against the perpetrator. Friends also show empathy and are less likely to blame survivors (Ahrens \& Campbell, 2000). Friends report feeling validated by helping the survivor, with two-thirds of a college sample reporting that they had provided good support to a sexually assaulted friend (Banyard et al., 2010). Gender also plays a role; men are less certain of how to talk to the survivor and provide help than women (Ahrens \& Campbell, 2000; Banyard et al., 2010) but female friends report more distress (Banyard et al., 2010). Male students are also more likely to blame the survivor than female college students (Ahrens \& Campbell, 2000). Friends' own victimization history also has an impact on friends. For example, friends who have been victimized themselves report more emotional distress (Banyard et al., 2010), blame victims less, and believe that violence has a greater impact on the survivor than friends who have no victimization history (Ahrens \& Campbell, 2000). Friends are therefore impacted by violence, but less negatively than family members or romantic partners.

\section{Friends of IPV survivors}

Little research exists on friends of IPV survivors. One qualitative study examined how IPV impacted others (two thirds of participants were friends of survivors) (Latta \& Goodman, 
2011). Salient aspects of that experience were the importance of closeness with the survivor and whether friends felt responsible to intervene. Furthermore, friends were often disappointed that the survivor did not leave the relationship or listen to advice. Friends also felt torn between trying to limit the impact of violence on their lives and feeling guilty about not helping the survivor enough. Consistent with studies of SA survivors, participants reported distress and confusion in how to help or engage with the survivor (Latta \& Goodman, 2011). This qualitative work therefore suggests that although there is important overlap in the experience of IPV and SA friends, such as distress and confusion, IPV may uniquely impact friends, for example by making them feel responsible to intervene.

\section{Current study}

Until now, no quantitative studies have examined how IPV impacts friends of survivors. Because IPV and SA have certain overlapping characteristics, SA studies can inform this research. For example, both IPV and SA have mostly male perpetrators and female survivors. In addition, survivors of both types of violence may experience stigma and shame following victimization and take a long time to recover. Also, friends of IPV and SA survivors report a similar impact of violence, such as confusion and distress (Ahrens \& Campbell, 2000; Latta \& Goodman, 2011).

To understand how disclosure of sexual assault impacts friends of survivors, Ahrens and Campbell (2000) used victimization perspective theory to develop a measure called Impact on Friends. According to this theory, friends believe that they should help the survivor deal with their unwanted sexual experience. If friends feel unsuccessful in that role, they often feel frustrated and helpless (Ahrens \& Campbell, 2000). The Impact on Friends measure was shortened and further developed by Banyard et al. (2010) and administered to a sample of 
college students. They found three dimensions: Ineffectiveness/Confusion, Emotional Distress and Validation. Both Banyard et al. (2010) and Ahrens \& Campbell (2000) studied friends of SA survivors. In this study, I modify the Impact on Friends measure with the qualitative information from the study of friends of IPV and add four items specific to IPV (Latta and Goodman, 2011). The purpose is to develop a measure appropriate for friends of IPV survivors: to examine whether IPV and SA have a similar impact on friends; and to assess how disclosure of IPV affects the relationship between survivor and friend. I also examine whether participant gender and participant victimization history moderate impact on friends.

Because IPV and SA share certain characteristics, I expect a similar factor structure to emerge for the Impact on Friends-IPV as in the Impact on Friends measure for SA (Banyard et al. (2010). However, I also expect the four IPV items to form their own factor, since they address concerns that are specific to IPV, not SA. I also expect relationship quality to remain similar before and after the disclosure, as was the case for Ahrens and Campbell (2000). I expect gender to moderate results such that women will report more Emotional Distress and Validation, but men to report more Ineffectiveness/Confusion, as in Banyard et al. (2010). I also expect victimization to moderate impact, such that victimized participants will report more Emotional Distress than non-victimized participants, as in Banyard et al. (2010).

Finally, to establish predictive validity of the measure and more precisely map the impact of a friend disclosing IPV, I measure psychological symptoms of the friend. The three variables measured are depression symptoms, post-traumatic stress disorder (PTSD) symptoms and problem drinking. All of these problems are common in survivors of violence (Golding, 1999; Nemeroff et al., 2006; Plichta, 2004; Temple, Weston, Stuart \& Marshall, 2008; White \& Chen, 2002), so if violence does have a radiating impact, we may see similar problems in friends. Also, 
friends of survivors may use alcohol to cope with their distress (Kilpatrick et al., 2007). Because Emotional Distress and Ineffectiveness/Confusion are both negative impact factors, I expect them to predict increased psychological symptoms. Also, because Validation indicates positive impact, I expect that factor to predict decreased psychological symptoms. 


\section{METHOD}

\section{Participants}

Participants were recruited from Amazon Mechanical Turk (Mturk), an online survey service (Buhrmester, Kwang \& Gosling, 2011). Members from all over the world complete tasks for money, such as responding to questionnaires or categorizing materials that are posted on the site. Collecting data in this way is common in social sciences, especially Social Psychology. The benefits to collecting data through Mturk are that data can be collected fairly quickly and inexpensively. Buhrmester, Kwang \& Gosling (2011) found that Mturk users are quite similar to the general population sample of internet users and significantly more diverse than college samples. They also found that reliable data can be gathered quickly.

Table 1 shows demographics of participants. 951 participants were originally recruited, and $382(40.17 \%)$ excluded from analysis. Most of these participants were excluded because they did not correctly answer quality control items. Online surveys often incorporate quality controls, where an item will simply state: "To monitor data quality, please select 'Strongly agree' for this question." Those that do not choose the correct option may not be reading the survey carefully or paying enough attention for the data to be of high quality. In this study, two such items were incorporated and those that did not answer correctly were excluded. Other criteria for exclusion were not giving information about whether a friend has disclosed to them, not providing information about whether they were survivors themselves and not consenting to be included in the survey. Although 40\% seems like a high number, excluding such large portions of the sample are common in online data collection (Goodman, Cryder \& Cheema, 2012; Oppenheimer, Meyvis \& Davidenko, 2009). 
As seen in Table 1, 569 participants were used for analysis: 326 were male, 242 were female and 1 did not provide information on gender. Age ranged from 18 to 75 years old $(M=$ 29.82, $S D=9.20$ ), while 119 self-identified as White, 15 as Black, 8 as Hispanic, 396 as Asian, 3 as Pacific Islanders or Native Hawaiian, 5 as Alaska Native and 31 as other. Participants included 402 from India, 123 from the United States and 44 from a variety of other countries.

All participants answered questions about demographics and psychological symptoms. Participants in the Friends group (those who had a friend who had told them about IPV, N = 274) also answered questions about their relationship quality and the Impact on Friends-IPV measure. Participants in the Non-Friends group (those who did not have a friend who told them about IPV, $\mathrm{N}=295$ ) acted as a comparison group. Seventy-eight people in the Friends group and 18 people in the Non-Friends group were survivors of IPV themselves.

A comparison of included and excluded participants showed that excluded participants were significantly older, more likely to identify as Asian and were more likely to report being in India than included participants (although the number of Asians and Indians was still very high in the included group). Excluded participants were also more likely to have had their own history of victimization than included ones. Participants in the Friend and Non-Friend groups were also compared, which showed very similar differences to the previous comparison, except NonFriend were more likely to racially identify as 'Other.'

\section{Measures}

Impact on Friends-IPV. The Impact on Friends scale (Banyard et al., 2010) was modified for IPV and administered to Friends. The three subscales identified by Banyard et al. (2010) are Validation, Ineffectiveness/confusion and Emotional Distress. In addition to these, I wrote 4 new items which are specific to IPV, the first was: "I feel angry at the perpetrator," 
which was included because friends reported sometimes having to engage with the perpetrator and feeling angry (Latta \& Goodman, 2011). Friends of SA survivors have also reported anger at the perpetrator (Ahrens \& Campbell, 2000). The second new item was: "I felt responsible to intervene," as previous research shows that IPV may be a process that occurs over time and therefore it is possible to intervene in the violence (see Latta \& Goodman, 2011). The last two items were: "I was disappointed because my friend did not listen to me" and "I was disappointed that my friend put up with the violence," both of which were prominent among participants in the study by Latta and Goodman (2011). This item pool was submitted to a group of experts selected because of their experience with conducting research on violence who agreed that they should be included in the measure.

The reliability of the IOF measure for SA is 0.87 , but no data were presented on validity (Banyard et al., 2010). To assess predictive validity of the IOF-IPV, I tested whether those who report significant impact of the disclosure on their lives are also more likely to experience various psychological adjustment problems. Similarly, a relationship between positive impact and improved psychological adjustment would also indicate predictive validity of the measure. Because there is no other measure on this subject, measuring convergent validity is not possible.

To assess psychological symptoms, the following measures were administered:

Post-traumatic stress disorder. The short version of the post-traumatic screening scale consists of 7 items that ask about specific symptoms of PTSD. This measure has adequate reliability and validity both for clinical samples as well as the general population (Bohnert \& Breslau, 2011).

Depression. The Center for Epidemiologic Studies Depression Scale (CESD-10; Andresen, Malmgren, Carter \& Patrick, 1994) was used to screen for depression symptoms. The 
measure consists of 7 items with a four point scale reflecting how often they experience the symptoms described. This is a shortened version of a 20 item CES-D instrument, which has good internal consistency in the general population (Cronbach's alpha=0.85) (Devins et al., 1984). This measure correlates highly with other measures of depression, such as the Beck Depression Inventory (Beck et al., 1961; Santor et al., 1995).

Problem drinking. The TWEAK (Russell, 1984) measures problem drinking. Each letter in the name corresponds to a certain type of symptom: tolerance, worry, eye opener, amnesia and cutting down on drinking. The measure consists of 6 questions about drinking behaviors in the past 30 days, which participants answer yes or no. Previous research has confirmed the adequate reliability and validity of this measure (Russell, 1994).

Other items were the following:

Relationship quality. Relationship quality was measured with two questions adapted from Ahrens \& Campbell (2000): Whether the relationship has changed or remained the same, and how close the participant feels to the survivor. The first question is answered on a five point Likert scale and the second question on a three point Likert scale.

Victimization history. To see whether the impact on friends differs between those who have and have not experienced violence themselves, participants were asked: "Has a friend of yours ever told you that he/she was a survivor of IPV?“"

Demographics. Participants were asked to give information about their gender, age, race and what country they are in.

Quality control. To ensure that participants are reading the questionnaire thoroughly, two items that measure quality were embedded into the survey. The first item, embedded within the Impact on Friends measure, is the following: "To monitor data quality, please select 
'Strongly agree' for this question." The second item was placed in the CESD depression scale:

“To monitor data quality, please select 'Rarely or none of the time for this question."” Such items may verify that participants are actually reading the items (Meade \& Craig, 2012), and the data from those who have not chosen the correct option are excluded from analysis. Studies have found that incorporating such controls can improve online data quality (Meade \& Craig, 2012).

\section{Procedure}

Participants were recruited to complete a brief online survey. Users of the Mturk website read a brief project description and could decide whether they wanted to participate or not. Participants were presented with a definition of IPV and asked whether a friend had ever disclosed such an experience. The definition used was: "Intimate partner violence is a pattern of purposeful coercive behaviors that may include inflicted physical injury, psychological abuse, sexual assault, progressive social isolation, stalking, deprivation, intimidation and threats. These behaviors are perpetrated by someone who is, was or wishes to be involved in an intimate or dating relationship with an adult or adolescent survivor and are aimed at establishing control of one partner over the other" (Family Violence Prevention Fund, 1999). The Friends group gave information about demographics, whether they were survivors themselves, relationship quality, answered the Impact on Friends-IPV measure as well as completed measures of PTSD, problem drinking and depression. The Non-Friends group gave information about demographics, whether they were survivors themselves as well as measures of PTSD, problem drinking and depression. Participants were paid 10 cents for their time. The average time it took participants to complete the survey was 5 minutes and 18 seconds. Before participating, Mturk users were asked whether they have reached the age of 18 and whether they consented to participate in the study. The study was approved by the University's Institutional Review Board. 


\section{Analysis}

Data from participants who did not answer quality control items correctly were removed from the sample $(\mathrm{N}=382)$. Next, a factor analysis was performed with the impact on friends items, which the Friends group (those who have had a friend disclose IPV) had answered. Exploratory Factor Analysis was performed to see whether the same three factor structure emerged with this sample, as in Banyard et al. (2010) and whether IPV-specific items loaded on those subscales. Factor analysis requires at least 10 participants per item (Cabrera-Nguyen, 2010), that condition was met here with 274 participants and 26 items. Psychological symptoms were computed for both groups to see whether the Impact on Friends measure predicts psychological outcomes. Demographic variables and own victimization history are also examined, to see whether any of these correlate with subscales of the Impact on Friends-IPV measure. The computer programs R (version 2.15.2) and SPSS (version 20) were used for these analyses. 


\section{RESULTS}

\section{Descriptive Information of Impact on Friends-IPV measure}

First, I examined descriptive information about items in the Impact on Friends-IPV (IOFIPV) measure, then I examine the factor structure of the measure, and finally I test the relationship of the factors to various psychological symptoms. Table 2 shows that the items most commonly endorsed (by at least $70 \%$ of participants) came from the Validation subscale of the Banyard et al. (2010) study, except for one item, which was IPV-specific. Those least commonly endorsed (by less than $30 \%$ of participants) came from the Ineffectiveness/Confusion subscale and one item from the Emotional Distress subscale of Banyard et al (2010). Less popular items may still be useful because around one-fourth of participants selected them. Therefore, most of the items are being used, potentially indicating that they reflect participants` experiences.

\section{Relationship quality}

The Friend group participants answered three items about the quality of the relationship between themselves and the survivor. The first item asked whether the relationship had gotten better, worse or stayed the same after disclosure. On a 5 point scale, most believe that the relationship stayed the same or got a little better $(M=3.21$ and $S D=1.01)$. This also fits with how close participants feel to the survivor, which before the disclosure was $M=2.39, S D=0.60$ and after it, $M=2.46, S D=0.61$, (on a three point scale), indicating a slight but non-significant increase in the positive direction. An average of 6-12 months had passed between the IPV and the disclosure, $(M=3.39, S D=1.54)$. Overall, these results suggest that some time had passed between the violence and the disclosure and that participants felt that their relationship was not negatively impacted. 


\section{Gender}

Women and men responded similarly, except for three items. When asked whether they felt burdened by their friend's needs, men $(M=2.97)$ reported that to be the case more often than women $(M=2.64), t=2.26, p=0.03$. Also, women $(M=4.11)$ reported more anger towards the perpetrator than men $(M=3.82), t=-2.29, p=0.02$. Finally, men $(M=3.64)$ reported more often than women $(M=3.41)$ that they felt they needed to become involved in efforts to combat IPV, $t=2.02, p=0.04$.

\section{Victimization}

Friends who are survivors and non-survivors differed on 12 items, as seen in Table 3 . The items come from all three subscales of the Banyard et al. (2010) measure: Items 5 and 13 came from Validation subscale, items 6, 15, 17 and 21 from the Ineffectiveness/Confusion subscale and items 11, 16, 20, 23 and 25 from the Emotional Distress subscale. Finally, item 14 was IPV specific. Survivors were more likely to endorse all of the items, except for item 14, about anger against the perpetrator.

\section{Psychological symptoms}

The psychological symptoms measured for this study were depression symptoms, posttraumatic stress disorder (PTSD) symptoms and problem drinking. However, analyses of problem drinking were not performed, as a large portion of the sample reported not drinking alcohol at all (36.87\%). There was a significant positive correlation between depression symptoms and PTSD symptoms, $r=0.57, p<0.001$.

Table 4 shows correlations between items of the IOF-IPV scale and psychological variables. The items vary in their relationships with depression and PTSD. A number of them have no significant relationship with psychological symptoms (items: 1, 3, 8, 10, 12, 13, 22, 24 
and 26). On the other hand, many other items are strongly correlated with depression and PTSD, suggesting that these items may be driving the relationship between factors and psychological variables. Breaking the scale down into items may be useful to see exactly what parts of the IOFIPV measure have an association with psychological symptoms.

I compared those who had a friend disclose violence with those who did not receive disclosures of violence. There were no significant differences for PTSD symptoms between friends of survivors $(M=2.57, S D=2.29)$ and non-friends of survivors $(M=2.86, S D=2.22)$, or on depression symptoms, (friends: $M=15.30, S D=4.99$, non-friends: $M=15.15, S D=4.48$ ). Next, I examined gender differences. Male friends $(M=15.96, S D=4.56)$ reported significantly higher depression symptoms than female friends $(M=14.40, S D=5.43), t(212.62)=2.46, p=$ $0.02, d=0.31$. No other gender differences were significant. There were therefore no differences between friends and non-friends, and men reported more depression symptoms than women.

I also tested for victimization differences for PTSD and depression symptoms, seen in Table 5. Survivors reported significantly greater depression symptoms than non-survivors, (friends: $t(265)=3.83, p<0.001$, non-friends: $t(288)=3.97, p<0.001)$. For PTSD symptoms, the same patterns was observed, with survivors reporting more symptoms than non-survivors (friends: $t(262)=3.57, p<0.001, d=0.47$, non-friends: $t(287)=2.50, p=0.01, d=0.30$ ). Survivors therefore report greater depression and PTSD symptoms than non-survivors.

Finally, I examined relationship quality by gender and victimization. Relationship quality did not differ by gender, but longer time had passed between IPV and disclosure for women $(M=$ 3.63, $S D=1.68)$ than for men $(M=3.23, S D=1.39), t(271)=-2.14, p=0.04, d=0.26)$. For victimization, less time elapsed between IPV and disclosure for survivors $(M=3.03, S D=1.50)$ than for non-survivors $(M=3.52, S D=1.52), t(270)=-2.41, p=0.02, d=0.29$. Survivors 
therefore waited longer to disclose to women and to those who have no personal experience of IPV.

\section{Factor analysis}

I performed an exploratory factor analysis on the items in the IOF-IPV scale to see whether the same structure would emerge as in Banyard et al (2010) and how IPV specific items would load onto that structure. The data collected is appropriate for factor analysis because a Bartlett's test of sphericity was significant, $\chi^{2}(325)=1649.46, p<0.001$ and the Kaiser-MeyerOlkin measure of sampling adequacy was 0.78 . First, I used parallel analysis to examine the structure of the data set, which suggested a five factor solution. Parallel analysis compares study data against random data to determine how many factors are present in the study data. Next, I ran a Principal Axis Factor analysis with promax rotation and five factors. Promax rotation is an oblique rotation, which was used because the constructs in the scale are likely to be correlated with each other (see Table 7). Also, oblique rotation is commonly used for factor analysis in the social sciences (Cabrera-Nguyen, 2010). Items that did not reach at least a 0.3 loading or loaded on more than 1 factor were excluded (a total of 7 items). The first three factors were similar to the Banyard et al. (2010) solution, roughly corresponding to the Emotional Distress, Validation and Confusion subscales. The subscales therefore seemed conceptually clear; reliability (Cronbach's alpha) ranged from 0.65-0.82. However, the fourth factor consisted of three items and the fifth factor of only two items and both of them had low reliability (around 0.40 ). This solution was not adequate because of the low reliability of the last two subscales and traditionally three items are considered a minimum for a factor (Cabrera-Nguyen, 2010).

Therefore, I ran a second factor analysis extracting four factors, see Table 7. Again, I used Principal Axis Factor Analysis and promax rotation, dropping cross-loading and non- 
loading items (total of 6 items, number 4, 5, 6, 7, 16 and 20). The first three factors were similar to the previous solution, with the fourth and fifth factors of the previous solution combined into one factor. When examining these latter items, all of them address concerns that are more oriented towards IPV than SA. Some of them were in the Banyard et al (2010) scale but some were the new IPV items composed for this study. The factors were therefore Emotional Distress $(\alpha=0.82)$, Validation $(\alpha=0.73)$, Confusion $(\alpha=0.65)$ and IPV $(\alpha=0.55)$. Table 6 shows the standardized factor loadings for all of the items on the four factors and that most of them clearly load onto one factor. The communality coefficient $\left(h^{2}\right)$ shows that the factors explain between 14 and $53 \%$ of the variance in each item, $M=34.54 \%$. A chi-square test of model fit was significant, $\chi^{2}(274)=195.79, p<0.001$.

The items in each factor seem to be conceptually similar and correspond well to the Banyard et al. (2010) solution, except the Ineffectiveness items did not load with the Confusion items. Three of the Ineffectiveness items, "Felt efforts didn't help", "Felt uncomfortable talking to him/her" and "Felt afraid I was causing more harm" instead loaded on the Emotional Distress factor, and the remaining two were dropped from the solution. The fourth factor, IPV, is a combination of new items and some from Banyard et al.'s study (2010), but all of them address concerns connected with IPV, such as own safety and feeling responsible to intervene.

Descriptive information and reliability of factors can be seen in Table 8. Although the last factor has low reliability, it is still higher than in the five factor solution and will therefore be retained. Table 9 shows the correlation between factors and how much variance each factor explains. All of the factors are significantly correlated. Together all of the factors explain $34 \%$ of the variance in the items. 


\section{Factor scores predicting psychological symptoms}

First, I tested for factor score differences by victimization. Friends who were survivors $(M=0.36)$ were higher on the Emotional Distress subscale than non-survivors, $(M=-0.16)$, $t(108.62)=3.90, p<0.001, d=0.74$. No other factor scores differed by victimization. There were no factor score differences by gender.

Next, I tested whether factors predict psychological symptoms. For depression symptoms, both the Emotional Distress factor, and the IPV factor predict increased depression symptoms, $\beta=2.21, p<0.001$ and $\beta=0.85, p=0.04$, respectively. The Validation and Confusion factors do not predict depression symptoms. When examining PTSD, three of the factors predict increased PTSD symptoms: Emotional Distress, $\beta=1.35, p<0.001, \mathrm{IPV}, \beta=$ 0.43, $p=0.01$ and Confusion, $\beta=0.31, p=0.08$ (although that relationship was marginally significant). Validation does not predict PTSD symptoms.

Using multiple regression to predict psychological symptoms revealed that only Emotional Distress predicted depression symptoms, $\beta=2.52, p<0.001$. For PTSD symptoms, Emotional Distress was still the only significant predictor, $\beta=1.47, p<0.001$. These results show that Emotional Distress best predicts psychological symptoms. Finally, Validation does not appear to have a significant protective effect on psychological symptoms.

\section{Mediated regression}

Because Emotional Distress was the only subscale that uniquely predicted depression, but IPV predicted depression on its own, I tested whether Emotional Distress acted as a mediator between IPV and depression. Emotional Distress fully mediates the relationship between IPV and Depression, CI 0.44-1.49. For PTSD symptoms, Emotional Distress fully mediated the relationship between IPV and PTSD symptoms (CI: 0.28-0.84). Emotional Distress also partially 
mediated the relationship between Confusion and PTSD symptoms (CI: 0.36-83). Emotional Distress therefore seems to be a predictor on its own and acts as a mediator between other subscales and psychological problems.

\section{Moderated regression}

Given that Emotional Distress is the only subscale that uniquely predicts both depression and PTSD, I tested whether victimization status changed that relationship. Own victimization did not moderate the relationship between Emotional Distress and PTSD symptoms or depression symptoms. 


\section{DISCUSSION}

The main purpose of this study was to develop a measure for the impact on friends of disclosure of IPV. An exploratory factor analysis of the items in the Impact on Friends-IPV scale revealed a similar factor structure as reported by Banyard et al. (2010): Emotional Distress, Validation and Confusion. In their study, they combined Confusion and Ineffectiveness subscales because they were highly correlated, but in this study, Ineffectiveness items either loaded onto the Emotional Distress scale or were dropped. In addition, the analysis showed a distinct factor for IPV, which consisted of a combination of items originally used by Banyard et al. (2010) and those added for this study, which were based on previous qualitative research (Latta \& Goodman, 2011). Because this solution was similar to the scale for sexual assault, there seems to be important overlap in how violence impacts friends, both for IPV and SA. However, there also are concerns unique to IPV, which may be why those items specific to IPV constitute their own factor.

All items were endorsed by at least a quarter of participants, indicating that they may reflect participants' experiences. In fact, some of the items were endorsed by up to $80 \%$ of participants, which is higher than that found by Banyard et al. (2010) (9-65\%). The items may therefore also reflect experiences of IPV friends, even though most of them were developed for friends of SA survivors. However, differences could also be attributed to different sampling, Banyard et al., (2010) used an in-person US college student sample with a mean age of 19.95, while most participants in this online study came from India and their mean age was 30 .

When asked about relationship with the survivor, most participants who had a friend who had disclosed violence reported that their bond stayed the same or got a little better as a result of the disclosure, as was expected. This suggests that friends do not feel a negative impact when 
survivors disclose IPV. These results are consistent with Ahrens and Campbell (2000), where participants reported little negative impact on the relationship and the positive aspects remained or improved slightly. This sustained relationship or a small positive change can therefore be seen both with IPV friends and SA friends.

Gender did not emerge as an important moderator for the Impact on Friends-IPV items. Consistent with Banyard et al.(2010), men felt more burdened by their friend's needs than women. However, in this study, men reported feeling that they should get involved in efforts to combat IPV, while Banyard et al.(2010) found no gender difference. Women also experienced more anger towards the perpetrator, possibly indicating that women empathize more with survivors than men do; this was a new item, so no comparison with previous research is possible. Additionally, Banyard et al. (2010) found differences on 10 items that did not emerge in this study, possibly indicating that men and women have greater differences in their attitudes towards SA than they do towards IPV. It is also possible that the difference can be attributed to the different samples used. The gender composition of the two samples was very similar, so that is unlikely to have contributed to these differences.

Participant victimization predicted different responses to several items. Friends who were survivors more often endorsed items about personal safety, such as "Became afraid for my own safety", "Felt a loss of sense of security" and "No longer felt the world was a safe place." They also were more likely to endorse items about Ineffectiveness, such as "Felt afraid I was causing more harm" and "Felt my efforts didn't help." Increased safety concerns of this group may stem from the disclosure reminding participants of their own IPV experience and invoke feelings of fear and uncertainty. Similarly, if participants did not get help when they were victimized, they might become pessimistic about their own ability to help a friend who has experienced IPV. 
Banyard et al. (2010) did not test for victimization differences on individual items, but found that friends who were survivors scored higher than non-survivors only on the Emotional Distress subscale. Perhaps this indicates that it can be somewhat traumatic to deal with a friend's experience when you yourself are a survivor.

Psychological symptoms measured were depression symptoms, post-traumatic stress disorder (PTSD) symptoms and problem drinking. However, analyses of problem drinking were not performed, as a large portion of the sample reported not drinking alcohol at all (36.87\%). This may be because a large number of participants in the sample are from India, where drinking is less accepted than in the Western world (Benegal, 2005).

Friends and Non-friends of survivors did not differ on depression and PTSD symptoms, so Friends did not exhibit more psychopathology than Non-Friends. Gender and victimization predicted depression symptoms, with male friends reporting greater symptoms than female friends, and friends who were survivors reporting greater depression and PTSD symptoms than non-survivors.

To establish predictive validity of the measure, I collected information about depression and PTSD symptoms of participants. Using simple regression, I found that both the IPV subscale and Emotional Distress predict increased depression symptoms. Neither Confusion nor Validation are connected with depression. However, when all subscales were entered into a regression equation together, Emotional Distress was the only subscale that still predicted depression symptoms. In fact, Emotional Distress fully mediated the relationship between IPV and depression, suggesting that IPV may lead to distress, which then predicts depression, but it is not the IPV items themselves that are directly connected with depression symptoms. 
A similar pattern emerged with PTSD symptoms, where Emotional Distress, IPV and Confusion all predict increased PTSD symptoms. Validation had no relationship with PTSD symptoms. As with depression, Emotional Distress was the only subscale that uniquely predicted PTSD symptoms. In fact, Emotional Distress fully mediated the relationship between IPV and PTSD symptoms and partially mediated the connection between Confusion and PTSD symptoms. These results may mean that the effects of violence are radiating from the survivor to others, as previous research has suggested (Riger et al., 2002). In the future, more psychological variables should be tested, such as life satisfaction, preferably both before and after the disclosure, to more precisely map the impact of a friend disclosing IPV victimization.

Finally, I examined whether the impact could be explained with victimization of the friend, but found that victimization status did not moderate the relationship between Emotional Distress and the psychological variables (depression and PTSD). This suggests that being friends with an IPV survivor may be connected with psychological symptoms above and beyond being a survivor yourself.

These results show that the Emotional Distress subscale is the most useful one to predict psychological problems. IPV also predicts increased depression and PTSD symptoms, and for both of them, that effect works through Emotional Distress. Validation does not seem have a protective effect against psychological problems.

This study is limited because the data was collected from a convenience sample with limited race variation and included self-report and retrospective methods. The gender of the survivor is also unknown, which may influence how women and men react to and are affected by IPV. Also, in an effort to keep the survey short, short versions of psychological measurements were used, and relationship quality only assessed with three items. Administering longer and 
more precise measurements in the future will probably be useful to help understand more about how the influence of IPV radiates to others.

The greatest limitation of this study is perhaps that most participants come from India. It is therefore possible that the results here are specific to that country or culture. There are several aspects of family life and gender roles that are different between India and the United States. For example, extended families in India traditionally live together (often three to four generations under the same roof) (Kumari, 2009) and arranged marriages are common (Nanda, 1998). These may have implications for how IPV is defined and addressed. For example, hiding IPV from relatives is probably more difficult when you live with them. Parents may also feel more responsible to intervene if their child's partner is being abusive when the parents have arranged the marriage themselves. IPV may therefore be seen as more of a family or a community issue than just between intimate partners, as is common in the Western countries. Finally, because male privilege and hierarchy is accepted within Indian culture (Derne, 1994), IPV may be seen as normal (especially non-violent, controlling aspects). All of these factors may have influenced how participants responded to the questionnaire.

Also, because data were collected online, the questionnaire may only have reached those who are wealthy enough to have a computer and internet access. The generalizability of the measure is therefore limited. However, a similar factor structure to Banyard et al. (2010) was found, suggesting that the sampling may not have drastically influenced the results. Another limitation is that even though factors seem conceptually clear and the reliability of the scale is adequate, explained variance is not very high and model fit could be better. Therefore, there is room for improvement of this measure. Possibly this measure does not tap into important aspects of being friends with survivors. For example, the measure does not assess whether the violence 
has impacted other aspects of the friend's life, such as work and school, or whether relationships between the friend and other people have been affected. Finally, the measure focuses on individual friends and survivors and does not try to assess attitudes or activity in the surrounding community about IPV. Disclosing violence and asking for help would presumably be more challenging in an environment that does not address IPV or try to provide support for its survivors. In the future, a confirmatory factor analysis would enable us to see whether the same structure would emerge with a different sample. Also, because explained variance was low, perhaps there are some aspects of disclosure that have not yet been included in the scale, but that may emerge through qualitative work with friends of survivors. In the meantime, this exploratory study has given us some indication of how IPV radiates to friends of survivors and shown that although there is significant overlap with the impact of SA on friends, IPV has a unique impact. 


\section{CITED LITERATURE}

Ahrens, C. E., Cabral, G. \& Abeling, S. (2009). Healing or Hurtful: Sexual Assault Survivors' Interpretations of Social Rections From Support Providers. Psychology of Women Quarterly, 33(1), 81-94.

Ahrens, C. E. \& Campbell, R. (2000). Assisting Rape Survivors as They Recover From Rape: The Impact on Friends. Journal of Interpersonal Violence, 15(9), 959-986.

Andresen, E. M., Malmgren, J. A., Carter, W. B. \& Patrick, D. L. (1994). Screening for depression in well-older adults: Evaluation of a short form of the CES-D (Center for Epidemiologic Studies Depression Scale. American Journal of Preventive Medicine, 10(2), 77-8-4.

Banyard, V. L., Moynihan, M. M., Walsh, W.A., Cohn, E. S. \& Ward, S. (2010). Friends of Survivors: The Community Impact of Unwanted Sexual Experiences. Journal of Interpersonal Violence, 25(2), 242-256.

Beck, A. T., Ward, C. H., Mendelson, M., Mock, J., \& Erbaugh, J. (1961). An inventory for measuring depression. Archives of general psychiatry, 4(6), 561.

Benegal, V. (2005). India: Alcohol and Public Health. Addiction, 100(8), 1051-56.

Boehnert, K. M. \& Breslau, N. (2011). Assessing the performance of the short screening scale for post-traumatic stress disorder in a large nationally representative survey. International Journal of Methods in Psychiatric Research, 20(1), 1-5.

Budde, S. \& Schene, P. (2004). Informal Social Support Interventions and their Role in Violence Prevention: An Agenda for Future Evaluation. Journal of Interpersonal Violence, 19(3), 341-355. 
Buhrmester, M., Kwang, T. \& Gosling, S. D. (2011). Amazon’s Mechanical Turk: A New Source of Inexpensive, Yet High-Quality Data? Perspectives on Psychological Science, $6(1), 3-5$.

Cabrera-Nguyen, P. (2010). Author Guidelines for Reporting Scale Development and Validation Results in the Journal of the Society for Social Work and Research. Journal of the Society of Social Work and Research, 1(2), 99-103.

Campbell, R., Ahrens, C. E., Sefl, T., Wasco, S. M. \& Barnes, H. E. (2001). Social Reactions to Rape Survivors: Healing and Hurtful Effects of Psychological and Physical Health Outcomes. Violence and Survivors, 16(3), 287-302.

Campbell, R. S., Sullivan, C. M. \& Davidson, W. S. II (1995). Women who use domestic violence shelters: Changes in depression over time. Psychology of Women Quarterly, $19(2), 237-255$.

Carlson, B. E., McNutt, L. A., Choi, D. Y. \& Rose, I. M. (2002). Intimate Partner Abuse and Mental Health: The Role of Social Support and Other Protective Factors. Violence Against Women, 8(6), 720-745.

Coker, A. L., Smith, P. H., McKeown, R. E. \& King, M. L. (2000). Frequency and correlates of intimate partner violence by type: Physical, sexual, and psychological battering. American Journal of Public Health, 90 (4), 55-559.

Coker, A. L., Smith, P. H., Thompson, M. P., McKeown, R. E., Bethea, L. \& Davis, K. E. (2002). Social Support Protects against the Negative Effects of Partner Violence on Mental Health. Journal of Women's Health \& Gender-Based Medicine, 11(5), 465-476. 
Coker, A. L., Watkins, K. W., Smith, P. H. \& Brandt, H. M. (2003). Social support reduces the impact of partner violence on health: Application of structural equation models. Preventive Medicine, 37(3), 259-267.

Davis, R. C. \& Brickman, E. (1996). Supportive and Unsupportive Aspects of the Behavior of Others Towards Survivors of Sexual and Nonsexual Assault. Journal of Interpersonal Violence, 11(2), 250-262.

Derne, S. (1994). Hindu men talk about controlling women: Cultural ideas as a tool of the powerful. Sociological Perspectives, 37 (2), 203-227.

El-Bassel, N., Gilbert, L., Rajah, V., Folleno, A., \& Frye, V. (2001). Social support among women in methadone treatment who experience partner violence: Isolation and male controlling behavior. Violence Against Women, 7(3), 246-275.

Family Violence Prevention Fund. (1999). Preventing domestic violence: Clinical guidelines on routine screening. San Francisco: Family Violence Prevention Fund.

Filipas, H.H. \& Ullman, S.E. (2001). Social reactions to survivors of sexual assault from social network members. Violence and Survivors, 16, 673-692.

Follingstad, D. R., Brennan, A. F., Hause, E. S., Polek, D. S., \& Rutledge, L. L. (1991). Factors moderating physical and psychological symptoms of battered women. Journal of Family Violence, 6(1), 81-95.

Golding, J. M. (1999). Intimate partner violence as a risk factor for mental disorders: A metaanalysis. Journal of Family Violence, 14(2), 99-132.

Goodkind, J. R., Gillum, T. K. Bybee, D. I. \& Sullivan, C. M. (2003). The Impact of Family and Friends' Reactions on the Well-Being of Women With Abusive Partners. Violence Against Women, 9(3), 347-343. 
Goodman, L., Dutton, M. A., Weinfurt, K. \& Cook, S. (2003). The Intimate Partner Violence Strategies Index: Development and Application. Violence Against Women, 9(2), 163-186.

Goodman, L. A., \& Epstein, D. (2008). Listening to battered women: A survivor-centered approach to advocacy, mental health, and justice. Washington, DC: American Psychological Association.

Goodman, J. K., Cryder, C. E. \& Cheema, A. (2012). Data Collection in a Flat World: The Strengths and Weaknesses of Mechanical Turk Samples. Journal of Behavioral Decision Making, published online.

Kilpatrick, D. G., Acierno, R., Resnick, H. S., Saunders, B. E., \& Best, C. L. (1997). A 2-year longitudinal analysis of the relationships between violent assault and substance use in women. Journal of Consulting and Clinical Psychology, 65, 834 - 847.

Krishnan, S. P., Hilbert, J. C., \& VanLeeuwen, D. (2001). Domestic violence and help seeking behaviors among rural women. Family and Community Health, 24(1), 28-38.

Kumari, R. (2009). Globalizing India and domestic violence against women. In S. Shirwadkar (Ed.), Family Violence in India: Human Rights, Issues, Actions and International Comparisons (210-225). New Delhi, India: Rawat Publications.

Latta, R. E. \& Goodman, L. A. (2011). Intervening in Partner Violence Against Women: A Grounded Theory Exploration of Informal Network Members' Experiences. The Counseling Psychologist, 39(7), 973-1023.

Levendosky, A. A., Bogat, G. A., Theran, S. A., Trotter, J. S., von Eye, A., \& Davidson, W. S. (2004). The social networks of women experiencing domestic violence. American Journal of Community Psychology, 34(1/2), 95-109. 
Mancini, J. A., Nelson, J. P., Bowen, G. L., \& Martin, J. A. (2006). Preventing intimate partner violence: A community capacity approach. Aggression, Maltreatment, and Trauma, 13(3/4), 207-231.

Meade, A. W. \& Craig, S. B. (2012). Identifying careless responding in survey data. Psychological Methods, 17(3), 437-455.

Nanda, S. (2000). Arranging a marriage in India. In P. Devita, Stumbling Toward Truth: Anthropologists at Work (196-204). Prospect Heights, IL: Waveland Press.

Nemeroff, C. B., Bremner, J. D., Foa, E. B., Mayberg, H. S., North, C. S. \& Stein, M. B. (2006). Posttraumatic stress disorder: A state-of-the-science review. Journal of Psychiatric Research, 40(1), 1-21.

Oppenheimer, D. M., Meyvis, T., \& Davidenko, N. (2009). Instructional manipulation checks: Detecting satisficing to increase statistical power. Journal of Experimental Social Psychology, 45, 867-872.

Plichta, S. (2004). Intimate partner violence and physical health consequences: policy and practice implications. Journal of Interpersonal Violence, 19, 1296-1323.

Riger, S., Raja, S. \& Camacho, J. (2002). The Radiating Impact of Intimate Partner Violence. Journal of Interpersonal Violence, 17(2), 184-205.

Russell, M., Martier, S. S., Sokol, R. J., Mudar, P., Bottoms, S., Jacobson, S., \& Jacobson, J. (1994). Screening for Pregnancy Risk-Drinking. Alcoholism: Clinical and Experimental Research, 18(5), 1156-1161.

Rose, L. E., Campbell, J., \& Kub, J. (2000). The role of social support and family relationships in women's responses to battering. Health Care for Women International, 21(1), 27-39. 
Sackett, L. A., \& Saunders, D. G. (1999). The impact of different forms of psychological abuse on battered women. Violence and Victims, 14, 105-117.

Santor, D. A., Zuroff, D. C., Ramsay, J. O., Cervantes, P., \& Palacios, J. (1995). Examining scale discriminability in the BDI and CES-D as a function of depressive severity. Psychological Assessment, 7(2), 131-139.

Tan, C., Basta, J., Sullivan, C. M. \& Davidson II, W. S. (1995). The Role of Social Support in the Lives of Women Exiting Domestic Violence Shelters: An Experimental Study. Journal of Interpersonal Violence, 10(4), 437-451.

Temple, J. R. Weston, R., Stuart, G. L. and Marshall, L. L. (2008). The longitudinal association between alcohol use and intimate partner violence among ethnically diverse community women. Addictive Behaviors, 33, 1244-1248.

Thompson, M. P., Kaslow, N. J., Kingree, J. B., Rashid, A., Puett, R., Jacobs, D., et al. (2000). Partner violence, social support, and distress among inner-city African American women. American Journal of Community Psychology, 28(1), 127-143.

Ullman, S. E. (1996). Social reactions, coping strategies, and self-blame attributions in adjustment to sexual assault. Psychology of Women Quarterly, 20(4), 505-526.

Ullman, S. E. (1999). Social support and recovery from sexual assault: A review. Aggression and Violent Behavior: A Review Journal, 4(3), 343-358.

Ullman, S. E. (2000). Psychometric Characteristics of the Social Reactions Questionnaire. Psychology of Women Quarterly, 24, 257-271.

Ullman, S. E., \& Siegel, J. M. (1995). Sexual assault, social reactions, and physical health. Women's Health: Research on Gender, Behavior, and Policy, 1(4), 289-308. 
White, H., \& Chen, (2002). Problem drinking and intimate partner violence. Journal of Studies on Alcohol and Drugs, 64, 205-214. 
Table 1

Demographics for participants included and excluded from analysis as well as demographics for Friends and Non-friends

\begin{tabular}{|c|c|c|c|c|c|}
\hline & & $\begin{array}{l}\text { Included } \\
N=569\end{array}$ & $\begin{array}{c}\text { Excluded } \\
N=382\end{array}$ & $\begin{array}{l}\text { Friends } \\
N=274\end{array}$ & $\begin{array}{c}\text { Non-Friends } \\
N=295 \\
\end{array}$ \\
\hline \multirow[t]{2}{*}{ Age } & Mean & 29.82 & $28.04 * *$ & 30.13 & 29.53 \\
\hline & Standard deviation & 9.20 & 8.23 & 9.99 & 8.41 \\
\hline \multirow[t]{3}{*}{ Gender } & Female & $242(42.53)$ & $114(33.83)$ & $115(41.97)$ & $127(43.05)$ \\
\hline & Male & $326(57.29)$ & $181(53.71)$ & $158(57.66)$ & $168(56.95)$ \\
\hline & Missing & $1(0.18)$ & $42(12.47)$ & $1(0.36)$ & $0(0.00)$ \\
\hline \multirow[t]{7}{*}{ Race } & White & $119(20.62)$ & $27(8.74)$ & $66(23.74)$ & $53(17.73)$ \\
\hline & Black & $15(2.60)$ & $7(2.27)$ & $10(3.65)$ & $5(1.67)$ \\
\hline & Hispanic & $8(1.39)$ & $3(0.97)$ & $5(1.80)$ & $3(1.00)$ \\
\hline & Asian & $396(68.63)$ & $232(75.08)^{* *}$ & $185(66.55)$ & $211(70.57)^{*}$ \\
\hline & Pacific Islander/Native Hawaiian & $3(0.52)$ & $3(0.97)$ & $1(0.36)$ & $2(0.67)$ \\
\hline & Alaska Native/American Indian & $5(0.87)$ & $9(2.91)$ & $2(0.73)$ & $3(1.00)$ \\
\hline & Other & $31(5.37)$ & $28(9.06)$ & $9(3.24)$ & $22(7.36)^{*}$ \\
\hline \multirow[t]{4}{*}{ Country } & India & $402(70.65)$ & $265(78.64)^{* *}$ & $184(67.15)$ & $218(71.95)^{*}$ \\
\hline & United States & $123(21.62)$ & $13(3.86)$ & $70(25.55)$ & $53(17.49)$ \\
\hline & Other & $44(7.73)$ & $7(2.08)$ & $18(6.60)$ & $26(8.58)$ \\
\hline & Missing & $0(0.00)$ & $52(15.43)$ & $2(0.73)$ & $6(1.98)$ \\
\hline \multirow[t]{3}{*}{ Has a friend } & Yes & $274(48.15)$ & $140(41.54)$ & $274(100.00)$ & $0(0.00)$ \\
\hline & No & $295(51.85)$ & 155 (45.99) & $0(0.00)$ & $295(100.00)$ \\
\hline & Missing & $0(0.00)$ & $42(12.46)$ & $0(0.00)$ & $0(0.00)$ \\
\hline \multirow[t]{3}{*}{ Own victimization history } & Yes & $96(16.87)$ & $93(27.60)^{* *}$ & 78 (28.47) & $18(6.10)^{* *}$ \\
\hline & No & $473(83.13)$ & $200(59.35)$ & $196(71.53)$ & $277(93.90)$ \\
\hline & Missing & $0(0.00)$ & $44(13.06)$ & $0(0.00)$ & $0(0.00)$ \\
\hline
\end{tabular}

* difference is significant at the $\mathrm{p}<0.05$ level.

$* *$ difference is significant at the $\mathrm{p}<0.001$ level. 
Table 2.

Number and percentage of participants who agreed or strongly agreed with each item on the Impact on Friends Scale

\begin{tabular}{|c|c|c|}
\hline Items & $\begin{array}{c}\text { Number of } \\
\text { participants who } \\
\text { agreed or strongly } \\
\text { agreed }\end{array}$ & Percentage \\
\hline 1. I didn't know what to do & 140 & 51.10 \\
\hline 2. I was unsure what my friend needed & 136 & 49.60 \\
\hline 3. I felt responsible to intervene & 175 & 63.90 \\
\hline $\begin{array}{l}\text { 4. I was disappointed because my friend did not listen } \\
\text { to me }\end{array}$ & 94 & 34.30 \\
\hline 5. I felt at ease dealing with my friend's experience & 125 & 45.70 \\
\hline 6. I felt burdened by my friend's needs & 94 & 34.30 \\
\hline 7. I felt I wasn't supportive enough & 91 & 33.20 \\
\hline 8. I felt good about myself for helping & 210 & 76.60 \\
\hline 9. I became angry at society for IPV & 170 & 62.00 \\
\hline 10. I didn’t know enough & 97 & 35.40 \\
\hline 11. I became afraid for my own safety & 95 & 34.60 \\
\hline 12. I felt appreciated & 150 & 54.70 \\
\hline 13. I felt more knowledgeable about this problem & 195 & 71.20 \\
\hline 14. I felt angry at the perpetrator & 195 & 71.20 \\
\hline 15. I felt afraid I was causing more harm & 73 & 26.60 \\
\hline 16. I felt a loss of sense of security & 102 & 37.20 \\
\hline 17. I felt uncomfortable talking to him/her & 63 & 23.00 \\
\hline $\begin{array}{l}\text { 18. I felt like I needed to become involved in efforts } \\
\text { to deal with the broader problem of IPV }\end{array}$ & 165 & 60.20 \\
\hline 19. I felt upset dealing with my friend's experience & 167 & 60.90 \\
\hline 20. I no longer felt that the world was a safe place & 131 & 47.80 \\
\hline 21. I felt my efforts didn't help & 88 & 32.10 \\
\hline 22. I felt I was doing a good job helping my friend & 199 & 72.60 \\
\hline 23. I became afraid of being victimized & 90 & 32.80 \\
\hline $\begin{array}{l}\text { 24. I was disappointed that my friend put up with the } \\
\text { violence }\end{array}$ & 172 & 62.80 \\
\hline 25. I became afraid to do things I did before & 69 & 25.10 \\
\hline 26. I felt I was a good source of support for my friend & 219 & 80.00 \\
\hline
\end{tabular}


Table 3.

Victimization differences on the Impact on Friends-IPV measure

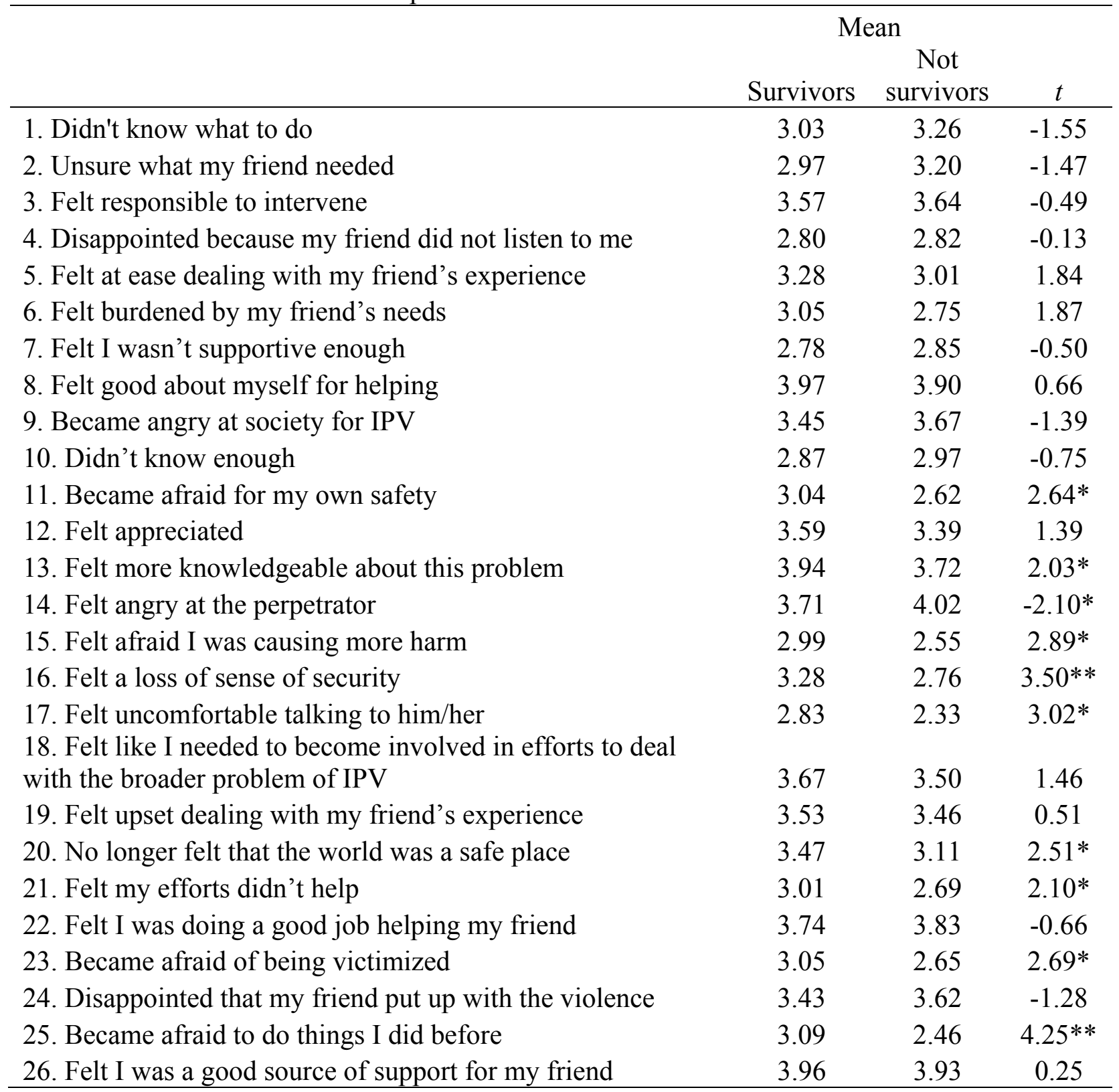

* Difference significant at the 0.05 level

** Difference significant at the 0.001 level 
Table 4

Correlation between IOF items and psychological symptoms

\begin{tabular}{lcc}
\hline & Depression & PTSD \\
\hline 1. I didn't know what to do & -0.01 & 0.02 \\
2. I was unsure what my friend needed & $0.16^{*}$ & $0.13^{*}$ \\
3. I felt responsible to intervene & 0.06 & 0.05 \\
4. I was disappointed because my friend did not listen to me & $0.18^{* *}$ & $0.23^{* *}$ \\
5. I felt at ease dealing with my friend's experience & $0.26^{* *}$ & $0.21^{* *}$ \\
6. I felt burdened by my friend's needs & $0.20^{* *}$ & $0.24^{* *}$ \\
7. I felt I wasn't supportive enough & $0.21^{* *}$ & $0.16^{*}$ \\
8. I felt good about myself for helping & 0.11 & 0.03 \\
9. I became angry at society for IPV & $0.18^{* *}$ & 0.05 \\
10. I didn't know enough & 0.01 & 0.07 \\
11. I became afraid for my own safety & $0.30^{* *}$ & $0.36^{* *}$ \\
12. I felt appreciated & 0.05 & -0.03 \\
13. I felt more knowledgeable about this problem & 0.04 & 0.09 \\
14. I felt angry at the perpetrator & $-0.18^{* *}$ & -0.11 \\
15. I felt afraid I was causing more harm & $0.19^{* *}$ & $0.37^{* *}$ \\
16. I felt a loss of sense of security & $0.24^{* *}$ & $0.39^{* *}$ \\
17. I felt uncomfortable talking to him/her & $0.16^{*}$ & $0.35^{* *}$ \\
18. I felt like I needed to become involved in efforts to deal with the & & \\
broader problem of IPV & $0.21^{* *}$ & $0.15^{*}$ \\
19. I felt upset dealing with my friend's experience & 0.09 & $0.15^{*}$ \\
20. I no longer felt that the world was a safe place & $0.27^{* *}$ & $0.35^{* *}$ \\
21. I felt my efforts didn't help & $0.23^{* *}$ & $0.28^{* *}$ \\
22. I felt I was doing a good job helping my friend & 0.01 & -0.05 \\
23. I became afraid of being victimized & $0.38^{* *}$ & $0.45^{* *}$ \\
24. I was disappointed that my friend put up with the violence & 0.09 & 0.11 \\
25. I became afraid to do things I did before & $0.37^{* *}$ & $0.44^{* *}$ \\
26. I felt I was a good source of support for my friend & 0.01 & -0.11 \\
\hline
\end{tabular}

* Difference significant at the 0.05 level

** Difference significant at the 0.001 level 
Table 5

Psychological symptoms by victimization

\begin{tabular}{lcccccc}
\hline & \multicolumn{2}{c}{ Friends } & \multicolumn{3}{c}{ Non-friends } \\
& $\begin{array}{c}\text { Survivors } \\
(N=78)\end{array}$ & $\begin{array}{c}\text { Non-survivors } \\
(N=196)\end{array}$ & \multicolumn{2}{c}{$\begin{array}{c}\text { Survivors } \\
(N=18)\end{array}$} & $\begin{array}{c}\text { Non-survivors } \\
(N=277)\end{array}$ \\
\hline Depression & 17.12 & 14.59 & $* *$ & 19.11 & 14.89 & $* *$ \\
PTSD & 3.34 & 2.24 & $* *$ & 4.11 & 2.77 & $*$ \\
\hline
\end{tabular}

* Difference significant at the 0.05 level

** Difference significant at the 0.001 level 
Table 6

Standardized factor loadings of items and how much variance of each item the factor solution explains $\left(h^{2}\right)$

\begin{tabular}{|c|c|c|c|c|c|}
\hline & $\begin{array}{c}\text { Emotional } \\
\text { Distress }\end{array}$ & Validation & Confusion & IPV & $h^{2}$ \\
\hline 1. Didn't know what to do & -0.10 & 0.18 & 0.79 & 0.03 & 0.48 \\
\hline 2. Unsure what my friend needed & 0.00 & 0.11 & 0.70 & 0.02 & 0.43 \\
\hline 3. Felt responsible to intervene & -0.11 & 0.10 & -0.03 & 0.37 & 0.14 \\
\hline $\begin{array}{l}\text { 4. Disappointed because my friend did } \\
\text { not listen to me }\end{array}$ & 0.28 & -0.06 & 0.13 & 0.07 & 0.15 \\
\hline $\begin{array}{l}\text { 5. Felt at ease dealing with my friend's } \\
\text { experience }\end{array}$ & 0.35 & 0.40 & -0.12 & -0.17 & 0.26 \\
\hline 6. Felt burdened by my friend's needs & 0.33 & -0.11 & 0.16 & 0.00 & 0.21 \\
\hline 7. Felt I wasn't supportive enough & 0.19 & -0.15 & 0.27 & 0.06 & 0.26 \\
\hline 8. Felt good about myself for helping & 0.03 & 0.59 & -0.09 & 0.12 & 0.42 \\
\hline 9. Became angry at society for IPV & 0.01 & 0.07 & -0.01 & 0.44 & 0.20 \\
\hline 10. Didn't know enough & 0.15 & -0.06 & 0.47 & -0.02 & 0.32 \\
\hline 11. Became afraid for my own safety & 0.68 & 0.07 & -0.06 & 0.04 & 0.45 \\
\hline 12. Felt appreciated & -0.01 & 0.61 & 0.17 & -0.11 & 0.29 \\
\hline $\begin{array}{l}\text { 13. Felt more knowledgeable about this } \\
\text { problem }\end{array}$ & 0.20 & 0.48 & -0 . & 0.04 & 0.29 \\
\hline 14. Felt angry at the perpetrator & -0.26 & 0.12 & 0.15 & 0.44 & 0.23 \\
\hline 15. Felt afraid I was causing more harm & 0.55 & -0.09 & 0.10 & 0.06 & 0.41 \\
\hline 16. Felt a loss of sense of security & 0.61 & -0.11 & -0.11 & 0.22 & 0.49 \\
\hline 17. Felt uncomfortable talking to him/her & 0.53 & -0.04 & 0.20 & -0.04 & 0.39 \\
\hline $\begin{array}{l}\text { 18. Felt like I needed to become involved } \\
\text { in efforts to deal with the broader } \\
\text { problem of IPV }\end{array}$ & 0.24 & 0.28 & -0.16 & 0.33 & 0.30 \\
\hline $\begin{array}{l}\text { 19. Felt upset dealing with my friend's } \\
\text { experience }\end{array}$ & 0.14 & -0.17 & 0.09 & 0.43 & 0.32 \\
\hline $\begin{array}{l}\text { 20. I no longer felt that the world was a } \\
\text { safe place }\end{array}$ & 0.40 & 0.00 & -0.28 & 0.42 & 0.42 \\
\hline 21. Felt my efforts didn't help & 0.39 & -0.21 & 0.18 & 0.00 & 0.35 \\
\hline $\begin{array}{l}\text { 22. Felt I was doing a good job of } \\
\text { helping my friend }\end{array}$ & -0.06 & 0.75 & 0.14 & 0.07 & 0.50 \\
\hline 23. Became afraid of being victimized & 0.77 & 0.01 & -0.13 & -0.05 & 0.53 \\
\hline $\begin{array}{l}\text { 24. I was disappointed that my friend put } \\
\text { up with the violence }\end{array}$ & 0.03 & 0.12 & 0.18 & 0.40 & 0.22 \\
\hline $\begin{array}{l}25 \text {. Became afraid to do things I did } \\
\text { before }\end{array}$ & 0.72 & 0.18 & 0.08 & -0.14 & 0.47 \\
\hline $\begin{array}{l}\text { 26. Felt I was a good source of support } \\
\text { for my friend }\end{array}$ & -0.11 & 0.71 & 0.24 & 0.20 & 0.45 \\
\hline
\end{tabular}


Table 7

Standardized factor loadings of items.

Factors

\begin{tabular}{|c|c|c|c|c|}
\hline Item & $\begin{array}{c}\text { Emotional } \\
\text { Distress }\end{array}$ & Validation & Confusion & IPV \\
\hline 23. Became afraid of being victimized & 0.78 & & & \\
\hline 25. Became afraid to do things I did before & 0.74 & & & \\
\hline 11. Became afraid for my own safety & 0.69 & & & \\
\hline 17. Felt uncomfortable talking to him/her & 0.54 & & & \\
\hline 15. Felt afraid I was causing more harm & 0.53 & & & \\
\hline 21. Felt my efforts didn't help & 0.36 & & & \\
\hline $\begin{array}{l}\text { 22. Felt I was doing a good job of helping my } \\
\text { friend }\end{array}$ & & 0.87 & & \\
\hline $\begin{array}{l}\text { 26. Felt I was a good source of support for my } \\
\text { friend }\end{array}$ & & 0.73 & & \\
\hline 8. Felt good about myself for helping & & 0.59 & & \\
\hline 12. Felt appreciated & & 0.50 & & \\
\hline 13. Felt more knowledgeable about this problem & & 0.37 & & \\
\hline 1. Didn't know what to do & & & 0.81 & \\
\hline 2. Unsure what my friend needed & & & 0.68 & \\
\hline 10. Didn't know enough & & & 0.51 & \\
\hline $\begin{array}{l}\text { 24. I was disappointed that my friend put up with } \\
\text { the violence }\end{array}$ & & & & 0.51 \\
\hline 14. Felt angry at the perpetrator & & & & 0.50 \\
\hline 3. Felt responsible to intervene & & & & 0.42 \\
\hline 19. Felt upset dealing with my friend's experience & & & & 0.39 \\
\hline 9. Became angry at society for IPV & & & & 0.39 \\
\hline $\begin{array}{l}\text { 18. Felt like I needed to become involved in } \\
\text { efforts to deal with the broader problem of IPV }\end{array}$ & & & & 0.34 \\
\hline
\end{tabular}


Table 8

Descriptive information for Impact on Friends subscales.

\begin{tabular}{llrlrr}
\hline Subscale & $\alpha$ & \multicolumn{1}{c}{$M$} & \multicolumn{1}{c}{$S D$} & \multicolumn{2}{c}{ Observed range } \\
\hline Emotional Distress & 0.82 & -0.01 & 0.91 & -2.17 & 2.71 \\
Validation & 0.73 & 0.00 & 0.91 & -3.64 & 2.15 \\
Confusion & 0.65 & 0.00 & 0.87 & -2.31 & 2.27 \\
IPV & 0.55 & 0.02 & 0.79 & -3.26 & 2.45 \\
\hline
\end{tabular}


Table 9

Correlation between factors

\begin{tabular}{lccccc}
\hline & & & & \multicolumn{2}{c}{$\begin{array}{c}\text { Proportion of } \\
\text { variance explained }\end{array}$} \\
\hline Emotional Distress & 1 & $-0.21^{* *}$ & $0.39^{* *}$ & $0.28^{* *}$ & 0.13 \\
Validation & - & 1 & $-0.53 * *$ & $0.22^{* *}$ & 0.09 \\
Confusion & - & - & 1 & $0.11^{*}$ & 0.05 \\
IPV & - & - & - & 1 & 0.07 \\
\hline
\end{tabular}

* Correlation is significant at the 0.05 level

** Correlation is significant at the 0.001 level 


\title{
INSTITUTIONAL REVIEW BOARD APPROVAL
}

\author{
UNIVERSITY OF ILLINOIS \\ AT CHICAGO
}

Office for the Protection of Research Subjects (OPRS)
Office of the Vice Chancellor for Research (MC 672)
203 Administrative Office Building
1737 West Polk Street
Chicago, Illinois 60612-7227

Approval Notice

Amendment to Research Protocol and/or Consent Document - Expedited Review UIC Amendment \# 1

March 11, 2013

Rannveig Sigurvinsdottir

Psychology

1007 W Harrison, M/C 285

Chicago, IL 60612

Phone: (312) 371-5715

RE:

Protocol \# 2012-0966

“The Impact of Intimate Partner Violence on Friends of Survivors"

Dear Ms. Sigurvinsdottir:

Members of Institutional Review Board (IRB) \#2 have reviewed this amendment to your research and/or consent form under expedited procedures for minor changes to previously approved research allowed by Federal regulations [45 CFR 46.110(b)(2) and/or 21 CFR $56.110(\mathrm{~b})(2)]$. The amendment to your research was determined to be acceptable and may now be implemented.

Please note the following information about your approved amendment:

Amendment Approval Date: $\quad$ March 4, 2013

Amendment:

Summary: UIC Amendment \#1 dated February 25, 2013, received February 27, 2013, is a investigator-initiated amendment to: 1) Revise the protocol showing changes in the method of data collection for the topic and to add measures to the survey used. Also, the PI abandoned collecting data from UIC students and to collect data through Amazon's Mechanical Turk. 2) There will be more measures added to the survey and a question will be added asking participants whether they themselves are IPV (Intimate Partner Violence) survivors or how they are impacted by having a friend who is an IPV survivor. Those who have a friend who is an IPV victim will complete demographic information, the Impact of Friends Measure and the three new measures of depression, post-traumatic stress disorder and problem drinking. Those who do not have a friend who is an IPV survivor will complete all of the same measures except the Impact on Friends scale. That group will therefore act as a comparison group, which will be especially useful for the measures of depression, post-traumatic stress disorder and problem drinking. The documents that are being revised are: the research protocol, a new informed consent form, a new debriefing form, a new subject information sheet, appendix Z (because the research is funded by the UIC Psychology department) and appendix 
$\mathrm{K}$ (because data will no longer be collected at UIC). The screening question will no longer be used and will be replaced with a recruitment posting.

Sponsor:

Research Protocol(s):

Department of Psychology

a) Impact on Intimate Partner Violence on Friends of Survivors Research Protocol; Version $2 ; 02 / 25 / 2013$

Recruiting Material(s):

a) Recruitment posting, Version 2, 3/9/13

Informed Consent(s):

a) Debriefing Form; Version 3; 02/25/2013

b) Informed Consent; Version 3;02/25/2013

Please note the Review History of this submission:

\begin{tabular}{|l|l|l|l|l|}
\hline Receipt Date & Submission Type & Review Process & Review Date & Review Action \\
\hline $02 / 27 / 2013$ & Amendment & Expedited & $03 / 04 / 2013$ & Approved \\
\hline
\end{tabular}

Please be sure to:

$\rightarrow$ Use only the IRB-approved and stamped consent document(s) and/or HIPAA Authorization form(s) enclosed with this letter when enrolling subjects.

$\rightarrow$ Use your research protocol number (2012-0966) on any documents or correspondence with the IRB concerning your research protocol.

$\rightarrow$ Review and comply with all requirements on the enclosure, "UIC Investigator Responsibilities, Protection of Human Research Subjects" (http://tigger.uic.edu/depts/ovcr/research/protocolreview/irb/policies/0924.pdf)

Please note that the UIC IRB \#2 has the right to ask further questions, seek additional information, or monitor the conduct of your research and the consent process.

Please be aware that if the scope of work in the grant/project changes, the protocol must be amended and approved by the UIC IRB before the initiation of the change.

We wish you the best as you conduct your research. If you have any questions or need further help, please contact the OPRS at (312) 996-1711 or me at (312) 355-2939. Please send any correspondence about this protocol to OPRS at $203 \mathrm{AOB}, \mathrm{M} / \mathrm{C} 672$.

Sincerely,

Jewell Hamilton, MSW

IRB Coordinator, IRB \# 2

Office for the Protection of Research Subjects

Enclosure(s):

1. Informed Consent Document(s):

a) Debriefing Form; Version 3; 02/25/2013

b) Informed Consent; Version 3; 02/25/2013

2. Recruiting Material(s): 
a) Recruitment posting, Version 2, 3/9/13

cc: $\quad$ Stephanie Riger, Faculty Sponsor, Psychology, M/C 285 Joe L. Martinez, Psychology, M/C 285 
VITA

\section{RANNVEIG SIGURVINSDOTTIR}

University of Iceland

B.Sc. in Psychology

2011

Honors Thesis: "Attitudes of Icelandic Police Officers towards

Intimate Partner Violence"

AWARDS

Fulbright scholar

$2011-2016$

TEACHING EXPERIENCE

University of Illinois at Chicago

Teaching assistant- Research methods in Psychology

Directed discussion sections, graded papers and met with

students.

Teaching assistant- Introduction to Psychology

2011-2012

Directed discussion sections, graded papers and met with students.

University of Iceland

Teaching assistant- Introduction to Psychology

2009-2010

Directed discussion sections and helped students.

\section{RESEARCH EXPERIENCE}

University of Illinois at Chicago

Research assistant

Worked on the Women's Stress and Support Study, a large

2012 - present

NIAAA funded study of sexual assault survivors.

Responsibilities included writing academic papers, mentoring undergraduate students, meeting and discussing with the research team as well as processing surveys, paying participants and conducting qualitative interviews. 


\section{PUBLICATIONS AND PAPERS}

\section{Conference presentations}

Sigurvinsdottir, R. \& Ullman, S. E. (2013). Differences in Sexual Risk Behaviors of Lesbian, Bisexual and Heterosexual Sexual Assault Victims. To be presented at the $69^{\text {th }}$ Annual Meeting of the American Society of Criminology in November 2013.

Sigurvinsdottir, R. (2013). Dating Violence: The Impact on Friends. Presented at the Biennial Conference of the Society for Community Research and Action (APA division 27) on June 27th 2013.

Sigurvinsdottir, R. \& Ullman, S. E. (2013). Social Reactions, Self-Blame and Problem Drinking for Sexual Assault Survivors. Presented at the Midwestern Psychological Association Conference on May $4^{\text {th }} 2013$.

Sigurvinsdottir, R. \& Ullman, S. E. (2013). Post-assault adjustment differences of sexual assault survivors by sexual orientation. Presented at the Chicago Graduate Student Research Symposium on April $5^{\text {th }} 2013$.

Sigurvinsdottir, R. \& Ullman, S. E. (2012). Self-Blame, Negative Social Reactions and Problem Drinking for Female Survivors of Sexual Assault. Presented at the Midwest EcologicalCommunity Psychology Conference on October 13 $3^{\text {th }}, 2012$.

Sigurvinsdottir, R. (2011). Attitudes of Icelandic Policemen Towards Interpersonal Violence. Presented at the 12th Conference of the International Academy of Investigative Psychology. Crime, Criminalistics and Criminal Psychology: New Directions in Investigative Behavioural Science on 31st March-2nd April 2011.

\section{Publications}

Sigurvinsdottir, R. \& Riger, S. (2012). The Criminal Justice Response to IPV: Problems and Possibilities. (Revise and resubmit in progress).

Sigurvinsdottir, R. \& Ullman, S. E. (2013). The Role of Sexual Orientation on Victimization and Recovery from Sexual Assault. (Under review.)

Ullman, S. E. \& Sigurvinsdottir, R. (2013). Intimate Partner Violence History and Drinking in Adult Sexual Assault Victims. (Under review.)

\section{PROFESSIONAL TRAINING}

Official, certified 40 hour training for domestic violence workers with the Chicago Metropolitan Battered Women's Network. Completed in January 2012. 